\title{
Evidence of Distributed Robust Surface Current Flow in 3D Topological Insulators
}

\author{
Janghee Lee, Jae-Hyeong Lee, Joonbum Park, Jun Sung Kim, and Hu-Jong Lee* \\ Department of Physics, Pohang University of Science and Technology, \\ Pohang 790-784, Republic of Korea \\ (Received 29 October 2013; revised manuscript received 19 January 2014; published 13 March 2014)

\begin{abstract}
The topologically protected conducting state is expected to exist on the entire surface of three-
\end{abstract} \\ dimensional topological insulators (TIs). Using concurrent measurements of the local and nonlocal \\ conduction, we provide experimental evidence for the topological robustness of the surface-conducting \\ states of bulk-insulating $\mathrm{Bi}_{1.5} \mathrm{Sb}_{0.5} \mathrm{Te}_{1.7} \mathrm{Se}_{1.3}$ crystalline flakes. The detailed investigation of local and \\ nonlocal charge conductance on the top surfaces, combining with the comprehensive numerical simulation, \\ reveals that the charge current is widely distributed over the entire surface of a TI. Our findings show \\ evidence of the presence of the topologically protected conducting state at the side wall with irregularly \\ stacked edges between the top and bottom surfaces. This study provides a reliable means of accurately \\ characterizing the topological surface states with inherent nonlocal surface-dominant conducting channels \\ in a TI.
}

DOI: 10.1103/PhysRevX.4.011039

Subject Areas: Condensed Matter Physics, Electronics, Topological Insulators

\section{INTRODUCTION}

Topological insulators (TIs) show new quantum states of matter whose surface supports metallic conducting channels, even with the Fermi level $\left(E_{F}\right)$ lying in the bulk energy gap [1-5]. The surface-conducting channels of a TI are topologically protected, as long as the time-reversal symmetry is preserved $[6,7]$. This is in sharp contrast to ordinary band insulators whose "accidental" surface state is not protected by physics. In addition, the nondegenerate helical spin texture of the topological surface-conducting state (TSS) [8,9] has attracted much attention, motivated by theoretical predictions of the emergence of exotic phenomena in the TSS, such as the magnetic monopole [10], topological exciton condensation [11], Majorana fermions [12], the quantum anomalous Hall effect [13], etc. The conduction nature of the TSS has been studied by tuning the $E_{F}$ well inside the bulk energy gap, which is essential for reducing the bulk conduction that hampers the observation of the phenomena specific to the TSS. Despite many efforts, however, it is still challenging to confirm the topologically protected conduction on the surface of a three-dimensional (3D) TI, with negligible conduction in the bulk [14-23]. In particular, the existence of the robust surface-conducting state on the rough sides of a TI crystal,

*hjlee@postech.ac.kr

Published by the American Physical Society under the terms of the Creative Commons Attribution 3.0 License. Further distribution of this work must maintain attribution to the author(s) and the published article's title, journal citation, and DOI. which has not been directly confirmed by transport measurements, is of great interest.

In general, the surface-conducting channels of a TI are admixed with the conducting channels formed in the bulk via the thermal activation among impurity levels, causing difficulties with accurately characterizing the surface conduction. Even with sufficiently suppressed bulk conduction, the existence of the robust surface conduction itself and the consequent distributed current flow on a TI makes the accurate estimation of the surface conductance and its precise characterization complicated and challenging. For the dominant surface conduction with a reduced bulk contribution, the current bias between the source and drain, arranged on the top surface of a TI, is not confined to the region between the two electrodes but flows over the entire TI surface. Because of this partial leakage of the bias current out of the source-drain region, the surface conductance cannot simply be determined in terms of the local source-drain potential difference $\left(V_{\mathrm{loc}}\right)$ and the bias current $\left(I_{\text {bias }}\right)$, as $I_{\text {bias }} / V_{\text {loc }}$. Thus, in particular, a significant error can arise in estimating the backgate-voltage dependence of the top surface conductance, which is hardly affected by the backgating, as the backgating can significantly affect the current-flow profile on the top surface. Despite the issue of this distributed current flow on a TI surface, it has never been taken into account in estimating the surface conductance of a TI. This may have been the cause of the inconsistency reported previously in transport properties of TIs, such as the wide sample dependence of the magnetoresistances (MR) [15,17,22-30] and the lack of consensus on the value of the Lande $g$ factor of electrons in the TSS [15,25,31-35]. 
In this study, we resolved the issue by concurrently monitoring the local $\left(V_{\text {loc }}\right)$ and nonlocal $\left(V_{\text {nloc1 }}, V_{\text {nloc } 2}\right)$ potential differences in $\mathrm{Bi}_{1.5} \mathrm{Sb}_{0.5} \mathrm{Te}_{1.7} \mathrm{Se}_{1.3}$ (BSTS) single crystalline flakes and matching them to numerical simulation [36] based on the finite-element method. With $E_{F}$ residing inside the bulk energy gap, owing to the electronhole carrier compensation in the material [37], we observed appreciable nonlocal signals. It is possible only in the presence of a robust conducting state, i.e., a TSS, over the entire surface of the crystal, including the sides, which are often rugged or rough with irregularly stacked edges between the top and bottom surfaces (see Appendix A). This distributed current flow, with a significant amount of current leaking into the bottom surface, suggests that the electrical surface conductance of a TI cannot be determined from local measurements $\left(V_{\text {loc }}\right)$ only. This study provides a direct confirmation of the presence of the TSS, even on the rough sides of a TI, and demonstrates that the surface conductance can be determined accurately only with concurrent measurements and a comprehensive analysis of both the local and nonlocal potential differences on the top surface.

\section{EXPERIMENTAL METHOD}

Single crystals of BSTS were grown using the self-flux method [25,38]. Stoichiometric mixtures of high-purity starting materials $[\mathrm{Bi}(5 \mathrm{~N}), \mathrm{Sb}(5 \mathrm{~N}), \mathrm{Te}(5 \mathrm{~N}), \mathrm{Se}(5 \mathrm{~N})]$ were loaded in an evacuated quartz ampoule, which was then heated up to $850^{\circ} \mathrm{C}$. After annealing at $850^{\circ} \mathrm{C}$ for two days to enhance the material homogeneity, the melt mixture was slowly cooled down to $500^{\circ} \mathrm{C}$ at a rate of $2{ }^{\circ} \mathrm{C} / \mathrm{h}$ for a week. Before complete furnace cooling, it was post annealed at $600^{\circ} \mathrm{C}$ for one more week to further improve the crystallinity. The stoichiometry and the high crystallinity of the single crystals were confirmed by the energy dispersive spectroscopy and the $\mathrm{x}$-ray diffraction, respectively. Recent scanning tunneling microscopy and spectroscopy on our BSTS crystals showed that their surface states have linear dispersion and chirality from spin texture, thus verifying the topological nature. This implies that topological surface states remain intact even with alloying of constituent atoms and significant surface disorder [39].

For transport measurements, a BSTS flake was mechanically exfoliated onto a heavily doped Si substrate, capped with a 300-nm-thick $\mathrm{SiO}_{2}$ layer [see Figs. 1(a) and 1(b)], which allowed gate tunability of the $E_{F}$ at the bottom surface of the BSTS flake. To prevent electrical shorting at the sides of the BSTS flake, we electrically insulated the side surface by covering the edge of the flake with crosslinked poly(methyl methacrylate) (PMMA) $950 \mathrm{k} \mathrm{C4}$ [inset of Fig. 1(a)] before thermally depositing the electrodes on the top. Electrical contacts were made using a standard electron (e)-beam lithography process followed by e-gun evaporation of $\mathrm{Ti} / \mathrm{Au}(10 \mathrm{~nm} / 300 \mathrm{~nm})$ electrodes. The
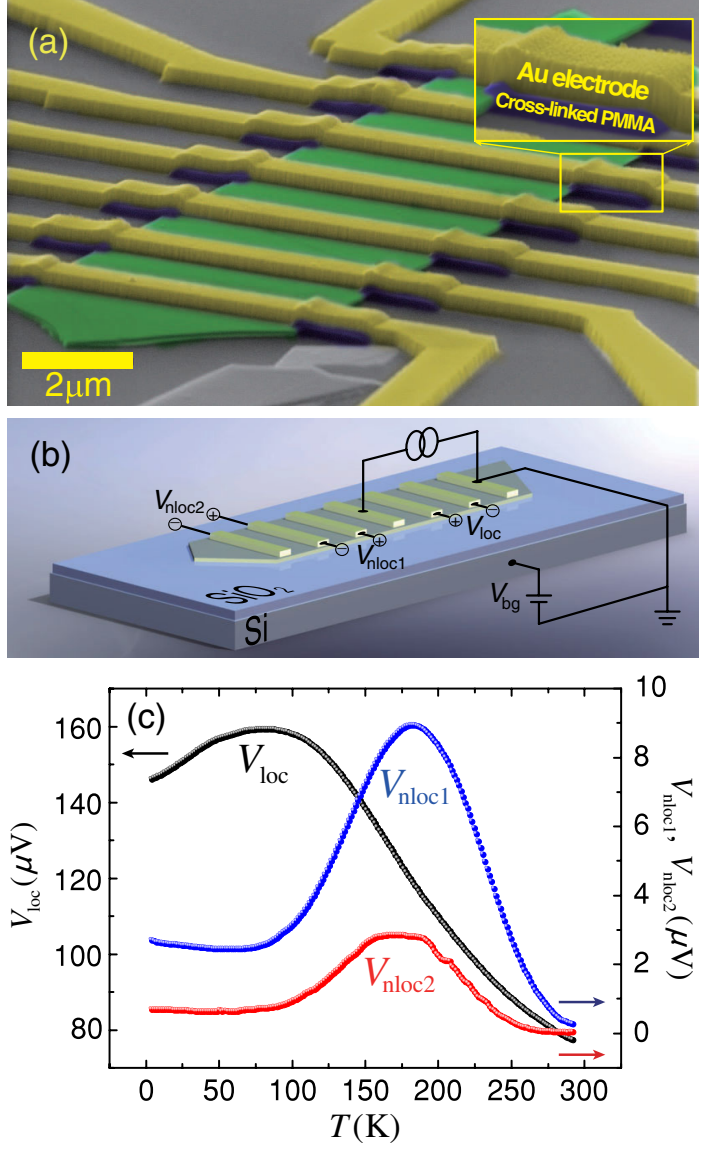

FIG. 1. (a) Scanning-electron-microscopy image of our BSTS thin flake, with Ti/Au (10 nm/300 nm thick) electrodes. The dark-blue regions under the $\mathrm{Au}$ electrodes (see the magnified view in the inset) are cross-linked PMMA insulating layers, which prevent shorting between the Au electrodes and the BSTS flake. The scale bar is $2 \mu \mathrm{m}$ in length. The sample dimensions are summarized in Table I. (b) Schematic diagram of the measurement configuration. (c) Temperature dependence of $V_{\text {loc }}$ (black line), $V_{\text {nloc1 }}$ (blue line), and $V_{\text {nloc2 }}$ (red line).

transport measurement was conducted via low-frequency lock-in techniques with a r.m.s. bias current of $100 \mathrm{nA}$ at 17.77 Hz. The details of the sample dimensions are summarized in Table I.

TABLE I. Sample dimensions. These parameters are used in numerical simulations.

\begin{tabular}{lc}
\hline \hline Physical parameters & Length \\
\hline Thickness of flake & $110 \mathrm{~nm}$ \\
Width of flake & $5.5 \mu \mathrm{m}$ \\
Width of electrodes & $0.8 \mu \mathrm{m}$ \\
Thickness of electrodes & $310 \mathrm{~nm}$ \\
Spacing between electrodes (edge to edge) & $1.9 \mu \mathrm{m}$ \\
\hline \hline
\end{tabular}




\section{RESULTS AND ANALYSIS}

\section{A. Observation of the nonlocal potential difference}

Figure 1(a) shows the scanning electron microscopy (SEM) image of a device. We measured $V_{\text {loc }}, V_{\text {nloc1 }}$, and $V_{\text {nloc2 }}$ concurrently, using the configuration shown in Fig. 1(b). The (+) and (-) signs in Fig. 1(b) indicate the polarities of the voltage probes. Figure 1(c) shows the temperature $(T)$ dependencies of $V_{\text {loc }}, V_{\text {nloc1 }}$, and $V_{\text {nloc2 }}$. Appreciable nonlocal signals $\left(V_{\text {nloc1 }}\right.$ and $\left.V_{\text {nloc2 }}\right)$ were observed; $V_{\text {nloc1 }}$ was evident, even at room temperature $\left[V_{\text {nloc } 1}(300 \mathrm{~K}) \approx 0.25 \mu \mathrm{V}\right]$. Similar behavior was also observed in another BSTS flake of this study. In a standard Hall-bar geometry, nonlocal resistance can appear because of the quantum spin Hall effect [40], the spin Hall effect [41], or the quasiballistic transport mechanism [42]. However, such causes are not applicable to our device without Hall-bar contacts [see Fig. 1(b)]. The most plausible cause of the observed nonlocal signals, $V_{\text {nloc1 }}$ and $V_{\text {nloc2 }}$, is the presence of conducting channels over the entire surface, including both the side and bottom surfaces, of the BSTS flake, which is more prominent when the conduction through the bulk is negligible. This feature is unique to the 3D TI but has not been directly confirmed by transport measurements. The simulation results, compared with the measured $V_{\mathrm{bg}}$ and magnetic-field dependencies of $V_{\text {loc }}, V_{\text {nloc1 } 1}$, and $V_{\text {nloc2 }}$, indicate that the observed finite nonlocal signals are a direct consequence of the current distribution, which was not confined between the source and drain but was widespread over the entire surface of the BSTS flake, with negligible current flow in its bulk. In addition, our experimental and simulation results commonly showed that $V_{\text {nloc1 }}$ and $V_{\text {nloc2 }}$ had similar $V_{\text {bg }}$ and magnetic-field dependencies, although they exhibited slight differences in their magnitudes.

\section{B. Numerical simulation method}

We performed numerical simulations on the current distribution, based on the finite-element method, using the commercial software package COMSOL [36]. Details of the validity of the numerical simulation are described in Appendix C. For the numerical simulation, we used the actual shape and dimensions of the device (see Table I), which were determined from atomic force microscopy and SEM. The sheet conductance of the top $\left(G_{\square}^{\text {top }}\right)$ and bottom $\left(G_{\square}^{\text {bot }}\right)$ surfaces depends on the extent of the band bending at the two surfaces $[8,43,44]$. In general, the values of $G_{\square}^{\text {top }}$ and $G_{\square}^{\text {bot }}$ are different. Therefore, numerical simulations were performed for different combinations of $G_{\square}^{\text {top }}$ and $G_{\square}^{\text {bot }}$, with each ranging from $0.3 e^{2} / h$ to $20 e^{2} / h$.

We also examined the influence of the bulk conductivity $\sigma_{\text {bulk }}$ on the values of $V_{\text {loc }}, V_{\text {nloc1 }}$, and $V_{\text {nloc2 }}$. Numerically simulated values of $V_{\text {nloc } 1}$, in Fig. 2(b), almost vanish for $\sigma_{\text {bulk }} \gtrsim 10 \mathrm{~S} / \mathrm{m}$ in our BSTS flake. Figure 2 also shows that
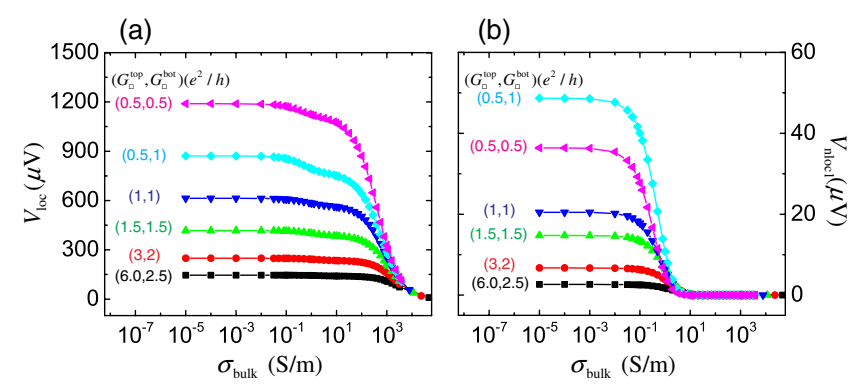

FIG. 2. Numerical simulation results of the effect of $\sigma_{\text {bulk }}$ on (a) $V_{\text {loc }}$ and (b) $V_{\text {nloc1 }}$ for various conductance pairs $\left(G_{\square}^{\text {top }}, G_{\square}^{\text {bot }}\right)$.

$V_{\text {loc }}$ and $V_{\text {nloc1 } 1}$ are almost independent of $\sigma_{\text {bulk }}$ when $\sigma_{\text {bulk }}<10^{-2} \mathrm{~S} / \mathrm{m}$ for all combinations of $G_{\square}^{\text {top }}$ and $G_{\square}^{\text {bot }}$, indicating that, in this case, the surface conduction predominates over the bulk conduction.

In our simulation, $\sigma_{\text {bulk }}$ is adopted in the range of $10^{-3} \sim 10 \mathrm{~S} / \mathrm{m}$. See Appendix $\mathrm{C}$ for details of the numerical simulation method.

\section{C. $V_{b g}$ dependence of local and nonlocal signals}

Figure 3(a) illustrates our simulation model. A bias current $\left(I_{\text {bias }}=100 \mathrm{nA}\right)$ was injected from the source to the drain. $S_{\text {loc }}$ and $S_{\text {nloc } 1}$ represent the cross sections where the total currents in the respective local and nonlocal $\left(V_{\text {nloc1 }}\right)$ regions are calculated. We also defined the horizontal bulk cross section $\left(S_{\text {bulk }}\right)$ in addition to the top $\left(S_{\text {top }}\right)$ and bottom $\left(S_{\text {bot }}\right)$ surfaces. Figure 3(b) shows how transport parameters are extracted by matching the experimental data to the simulation results. We calculated a set of $\left(V_{\text {loc }}, V_{\text {nloc1 }}\right.$, $V_{\text {nloc2 }}$ ) for each input parameter set of ( $\left.\sigma_{\text {bulk }}, G_{\square}^{\text {top }}, G_{\square}^{\text {bot }}\right)$. A few million sets of $\left(V_{\text {loc }}, V_{\text {nloc1 }}, V_{\text {nloc } 2}\right)$ for different combinations of input parameters $\sigma_{\text {bulk }}, G_{\square}^{\text {top }}$, and $G_{\square}^{\text {bot }}$ were prepared for matching between the data and the simulation results within uncertainty of $\left|\Delta V_{\text {loc }}\right| \leq 1 \mu \mathrm{V}$, $\left|\Delta V_{\text {nloc1 } 1}\right| \leq 0.3 \mu \mathrm{V}$, and $\left|\Delta V_{\text {nloc } 2}\right| \leq 0.05 \mu \mathrm{V}$, which gave rise to an optimum value set of $\left(\sigma_{\text {bulk }}, G_{\square}^{\text {top }}, G_{\square}^{\text {bot }}\right)$ corresponding to each data set. In this manner, from the $V_{\mathrm{bg}}$ dependence of $V_{\text {loc }}, V_{\text {nloc1 }}$, and $V_{\text {nloc2 }}$ [Fig. 3(c)], we obtained $R_{\square}^{\text {top }}\left(=1 / G_{\square}^{\text {top }}\right)$ and $R_{\square}^{\text {bot }}\left(=1 / G_{\square}^{\text {bot }}\right)$ for each $V_{\text {bg }}$ [Fig. 3(d)]. The measured $V_{\text {bg }}$ dependence of $V_{\text {loc }}$ in Fig. 3(c) has an opposite response to that of $V_{\text {nloc1 }}$ (as well as $\left.V_{\text {nloc2 } 2}\right)$ at $4.2 \mathrm{~K}$. As the sheet resistance of the bottom surface $\left(R_{\square}^{\text {bot }}\right)$ increases, the current $\left(I_{\text {top }}\right)$ on the top surface at $S_{\text {loc }}$ is expected to increase, resulting in an increase (decrease) of $V_{\text {loc }}\left(V_{\text {nloc1 }}\right.$ and $\left.V_{\text {nloc2 } 2}\right)$. By matching the simulation to the measured results, we confirmed that this qualitative reasoning is correct and that the opposite variation of $V_{\text {loc }}$ and $V_{\text {nloc1 }}$ with $V_{\mathrm{bg}}$ in Fig. 3(c) is due to the ambipolar nature of the conduction in the bottom surface.

Figure 3(d) shows the $V_{\mathrm{bg}}$ dependence of $R_{\square}^{\text {top }}\left(=1 / G_{\square}^{\text {top }}\right), R_{\square}^{\text {bot }}\left(=1 / G_{\square}^{\text {bot }}\right)$, and $R_{\square}^{\text {exp }}\left(=\frac{V_{\text {loc }}}{I_{\text {bias }}} \frac{W}{L}\right)$, where $L$ is the center-to-center distance of the two voltage probes 
of $V_{\text {loc }}$ and $W$ is the width of our flake. This $V_{\mathrm{bg}}$ dependence of $R_{\square}^{\text {bot }}$ clearly shows the ambipolar nature of the bottom-surface conductance arising from a Diracfermionic dispersion relation, a distinguishing characteristic of the TSS, with the Dirac point at $V_{\mathrm{bg}}=-10 \mathrm{~V}$. It is reminiscent of the $V_{\mathrm{bg}}$ dependence of the sheet resistance in graphene, showing a maximum at the Dirac point.

However, as shown in Fig. 3(d), $R_{\square}^{\text {top }}$ remains almost insensitive to backgating. It is apparent that, with a thickness of about $110 \mathrm{~nm}$ for our BSTS flake, the effect
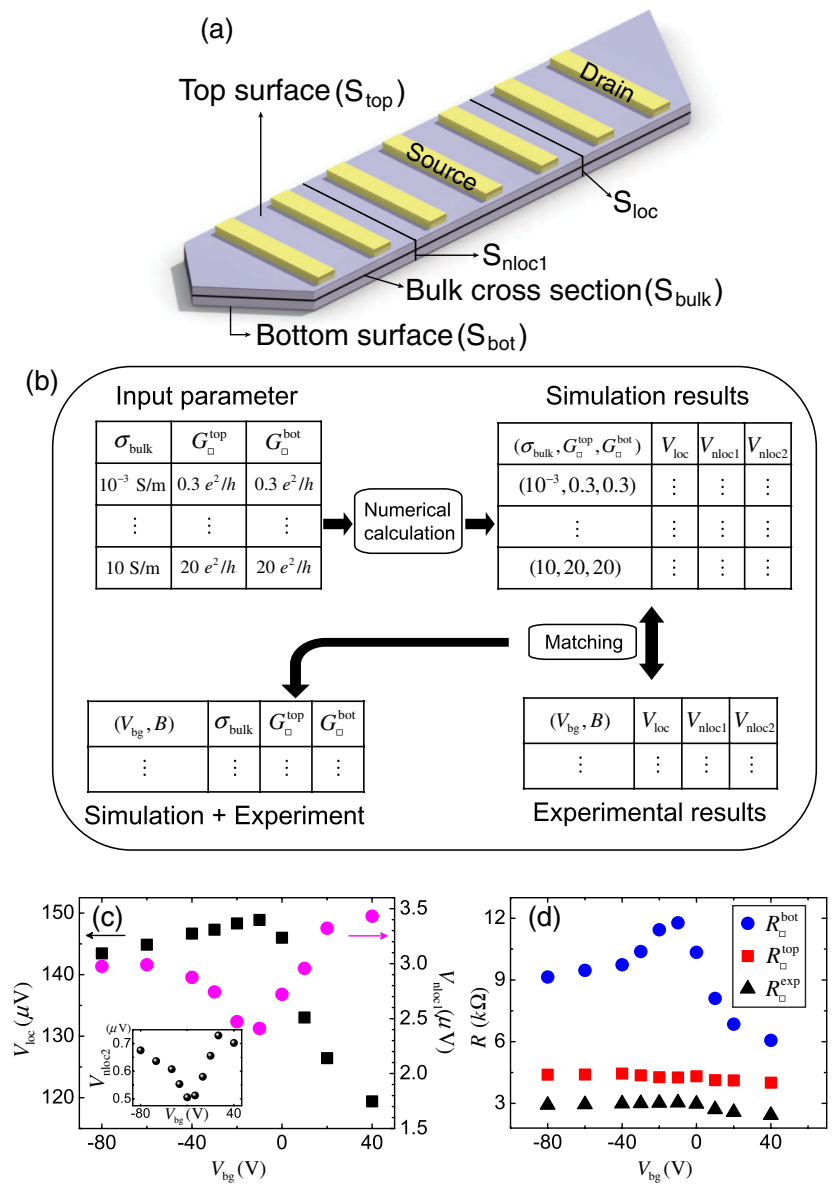

FIG. 3. (a) Simulation model (the thickness of BSTS is exaggerated for visual clarity). $I_{\text {bias }}=100 \mathrm{nA}$ is injected from the source to the drain. $S_{\text {loc }}\left(S_{\text {nloc1 }}\right)$ represents the hypothetical plane used to extract the total current through the cross-sectional area between the two voltage probes of $V_{\text {loc }}\left(V_{\text {nloc1 }}\right)$. (b) Schematic diagram for methodology of this study, illustrating how to determine an optimal set of values for $\left(\sigma_{\text {bulk }}, G_{\square}^{\text {top }}, G_{\square}^{\text {bot }}\right)$ by matching an observed set of $\left(V_{\text {loc }}, V_{\text {nloc } 1}, V_{\text {nloc2 }}\right)$ to the simulated results. (c) $V_{\text {bg }}$ dependence of $V_{\text {loc }}$ (black squares) and $V_{\text {nloc1 }}$ (magenta circles) taken at $4.2 \mathrm{~K}$. The inset shows $V_{\text {nloc2 }}$ vs $V_{\mathrm{bg}}$. (d) Simulation results of $R_{\square}^{\text {top }}\left(=1 / G_{\square}^{\text {top }}\right)$ and $R_{\square}^{\text {bot }}\left(=1 / G_{\square}^{\text {bot }}\right)$ matched to the measured $V_{\text {loc }}, V_{\text {nloc1 } 1}$, and $V_{\text {nloc2 }}$ simultaneously for each value of $V_{\mathrm{bg}} . R_{\square}^{\exp }\left(=\frac{V_{\text {loc }}}{I_{\text {bias }}} \frac{W}{L}\right)$ is also plotted for comparison, where $L$ is the center-to-center distance of the two voltage probes of $V_{\text {loc }}$ and $W$ is the width of our flake. of $V_{\mathrm{bg}}$ does not reach the top surface because of the screening at the bottom $[43,44]$. This is consistent with the results of our previous studies [45]. The resistance peak of $R_{\square}^{\text {bot }}$ in Fig. 3(d) has a value of around $2.2 e^{2} / h$. This value is about $1 / 4$ the minimum conductivity of graphene in the diffusive regime, with relatively low mobility (3000-8000 $\left.\mathrm{cm}^{2} / \mathrm{Vs}\right)$ [46], which may be consistent with the fact that the TSS has $1 / 4$ the degeneracy of graphene [47]. Thus, in summary, since $R_{\square}^{\text {bot }}$ increases as $V_{\mathrm{bg}}$ approaches the Dirac point, the currents at $S_{\mathrm{loc}}$ on the bottom surface and at $S_{\text {nlocl }}$ on the top surface decrease simultaneously, while the current at $S_{\text {loc }}$ on the top surface increases. Strikingly, the $V_{\mathrm{bg}}$ dependence of $V_{\text {loc }}$ and $V_{\text {nlocl }}$ in Fig. 3(c) is a consequence of variations in the current flow at $S_{\text {loc }}$ and $S_{\text {nloc1 }}$ on the top surface, rather than the change in resistance on the top surface.

$R_{\square}^{\exp }$, shown in Fig. 3(d) (triangles), is obtained from the observed value of $V_{\text {loc }}$ only, which is the conventional way of estimating the sheet resistance of samples. The relative discrepancy, $\left(\mid R_{\square}^{\text {exp }}-R_{\square}^{\text {top }}\right) \mid / R_{\square}^{\text {top }}$, between the "apparent" sample resistance taken on the top surface $\left(R_{\square}^{\exp }\right)$ and the true value of the top-surface resistance $\left(R_{\square}^{\text {top }}\right)$, reached $30 \%-40 \%$. A large discrepancy between $R_{\square}^{\text {exp }}$ and $R_{\square}^{\text {top }}$ (as well as $R_{\square}^{\text {bot }}$ ) indicates that the $R_{\square}^{\text {exp }}$ represents neither $R_{\square}^{\text {top }}$ nor $R_{\square}^{\text {bot }}$. This discrepancy is mainly caused by the distributed current flow over the entire TI surface and the difference in the values of $G_{\square}^{\text {top }}$ and $G_{\square}^{\text {bot }}$, together with their $V_{\mathrm{bg}}$ dependencies. Thus, the apparent conductance determined from the observed $V_{\text {loc }}$, without a comprehensive analysis including information on $V_{\text {nloc1 }}$ and $V_{\text {nloc2 } 2}$, will result in an erroneous estimation of the surface conductance and its gate dependence.

We also obtained bulk conductivity, which turned out to be highly sensitive to small fluctuations of $V_{\text {nloc2 }}$. Thus, only the upper limit of $\sigma_{\text {bulk }}$ was estimated meaningfully, at around $0.6 \mathrm{~S} / \mathrm{m}$. This value of $\sigma_{\text {bulk }}$ is a few tens of times smaller than the ones reported previously for extremely thick BSTS crystals (thickness of around $100 \mu \mathrm{m}$ ) $[25,38,48]$. Because the metallic surface-conducting channels always coexist with thermally activated bulkconducting channels in a 3D TI, the measured resistance represents the combined resistance of these conducting channels connected in parallel. The surface conductance amounts to at least a few $\mathrm{S} / \mathrm{m}$ [see Eq. (B4) in Appendix B]. Thus, if the measured bulk conductivity $\left(\sigma_{\text {bulk }}^{\text {exp }}\right)$ in thick crystals decreases below about $10 \mathrm{~S} / \mathrm{m}$, similar to the values obtained in previous reports on BSTS $[25,35,38,49-53], \sigma_{\text {bulk }}^{\text {exp }}$ can no longer represent the true bulk conductivity $\left(\sigma_{\text {bulk }}\right)$ of a 3D TI [see Appendix B for a discussion on the discrepancy between the measured apparent value $\left(\sigma_{\text {bulk }}^{\text {exp }}\right)$ and the true value $\left(\sigma_{\text {bulk }}\right)$ of bulk conductivity in a 3D TI]. For this reason, we suspect that the previously reported values of $\sigma_{\text {bulk }}^{\exp }$ of BSTS may have been significantly overestimated. 
We also show that the different $T$ dependencies among $V_{\text {loc }}$ and $V_{\text {nloc1,2 }}$ in Fig. 1(c), in the temperature range below about $70 \mathrm{~K}$ was caused by the combined $T$ dependencies of $G_{\square}^{\text {top }}, G_{\square}^{\text {bot }}$, and $\sigma_{\text {bulk }}($ see Appendix D).

\section{Distributed surface current flow}

Figures 4(a)-4(c) show the surface current profile using a common color code for $S_{\text {top }}, S_{\text {bulk }}$, and $S_{\text {bot }}$ of the simulation model [see Fig. 3(a)] for $\left(\sigma_{\text {bulk }}, G_{\square}^{\text {top }}, G_{\square}^{\text {bot }}\right)=$ $\left(0.06 \mathrm{~S} / \mathrm{m}, 6.0 e^{2} / h, 2.5 e^{2} / h\right)$, which corresponds to the observed values of $V_{\text {loc }}=146 \mu \mathrm{V}, V_{\text {nloc1 }}=2.72 \mu \mathrm{V}$, and $V_{\text {nloc2 }}=0.58 \mu \mathrm{V}$ at $4.2 \mathrm{~K}$ and $V_{\mathrm{bg}}=0 \mathrm{~V}$. The solid lines in Figs. 4(a)-4(c) are contour lines that show the current flow, perpendicular to the equipotential lines. Here, we assume vanishing off-diagonal components of the conductance tensor [54]. A significant current appears on the bottom surface [Fig. 4(c)]. However, the current density in the bulk almost completely vanishes [Fig. 4(b)]. Figures 4(d) and 4(e) show the $V_{\mathrm{bg}}$ dependence of the simulated total current on the top surface $\left(I_{\text {top }}\right.$; red squares), bottom surface ( $I_{\text {bot }} ;$ blue circles), two side surfaces ( $I_{\text {side }}$; black circles), and bulk ( $I_{\text {bulk }}$, magenta triangles) at $S_{\text {loc }}$. As expected, $I_{\text {bot }}$ becomes minimal at the Dirac point of the bottom surface, while $I_{\text {top }}$ and $I_{\text {side }}$ show the opposite $V_{\text {bg }}$ dependence. Even with the large cross-sectional area of the bulk, $I_{\text {bulk }}\left[=I_{\text {bias }}-\right.$ $\left.\left(I_{\text {top }}+I_{\text {bot }}+I_{\text {side }}\right)\right]$ is at most 3 pA [Fig. 4(e)].

$I_{\text {nloc1 }}$ (green squares) in Fig. 4(e) shows the nonlocal current on the top surface at $S_{\text {nloc1 } 1}$. In contrast to $I_{\text {bulk }}$, an appreciable amount of current appears on the top surface at
$S_{\text {nlocl }}$, with its direction opposite to that in the local-flow region. The large value of $V_{\text {nlocl }}$ in Fig. 1(c) results from this $I_{\text {nlocl }}$, which cannot be realized without the robust surface-conducting channels in our BSTS flake, in particular, at the side surfaces, a feature of the TI that is in sharp contrast to an ordinary band insulator. In fact, even for an ordinary band insulator, a surface-conducting state can arise from accidental causes (e.g., surface impurities, surface reconstruction, surface band bending, or band-gap narrowing near the surface). However, existence of these "accidental surface states" are not topologically robust. Thus, applying $V_{\mathrm{bg}}$ or, equivalently, shifting the $E_{F}$ can switch the accidental surface states on or off. However, as shown in Fig. 3(c), large values of $V_{\text {nlocl }}$ are observed for all $V_{\text {bg }}$, even with the ambipolar transport at the bottom surface in Fig. 3(d), which indicates the robustness of the surface-conducting state in our thin BSTS flake. In addition, if the top and bottom surfaces have opposite carrier types in an ordinary band insulator, a $p-n$ junction would form somewhere between the top and bottom surfaces and should suppress much of the nonlocal surface current, $I_{\text {nloc1 }}$. However, the observed nonvanishing $V_{\text {nloc1 }}$ in Fig. 3(c), irrespective of $V_{\mathrm{bg}}$, indicates that no such large conductance suppression develops on the sides of our BSTS flake (see Appendix A). Our observation of the robust and large $V_{\text {nloc1 }}$ cannot be explained in the absence of a gapless surface-conducting state, i.e., a Diracfermionic TSS. Thus, the side surfaces act as a filter that only passes the topologically protected surface currents contributing to the nonlocal potential difference.
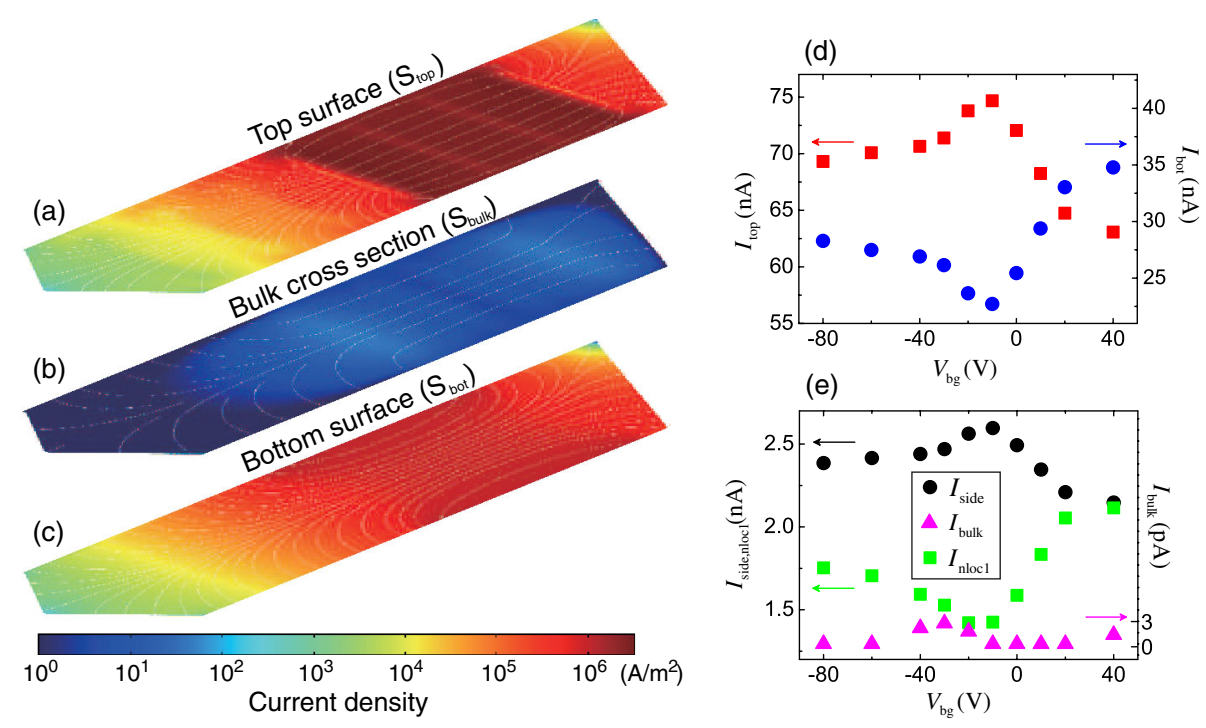

FIG. 4. (a),(b),(c) Numerical simulation results on the current distribution of our BSTS flake in Fig. 1(a). The current density profile at each plane, which is defined as in Fig. 3(a), is plotted using the same color scale for $\left(G_{\square}^{\text {top }}, G_{\square}^{\text {bot }}\right)=(6.0,2.5)\left(e^{2} / h\right)$ and $\sigma_{\text {bulk }}=0.06 \mathrm{~S} / \mathrm{m}$, which correspond to the observed values of $V_{\text {loc }}=146 \mu \mathrm{V}, V_{\text {nloc } 1}=2.72 \mu \mathrm{V}$, and $V_{\text {nloc } 2}=0.58 \mu \mathrm{V}$ at $4.2 \mathrm{~K}$ for $V_{\mathrm{bg}}=0 \mathrm{~V}$. The solid lines in (a), (b), and (c) are contour lines describing the current flow (i.e., these lines are perpendicular to the equipotential lines). (d) $V_{\text {bg }}$ dependence of $I_{\text {top }}$ (red squares) [ $I_{\text {bot }}$ (blue circles)], the current on the top (bottom) surface at the cross section of $S_{\text {loc }}$ depicted in Fig. 3(a). (e) $V_{\text {bg }}$ dependence of $I_{\text {side }}$ (black circles) [ $I_{\text {bulk }}$ (magenta triangles)], the current on the side surface (inside bulk) at the cross section $S_{\text {loc }}$ depicted in Fig. 3(a). $I_{\text {nloc } 1}$ is the nonlocal current on the top surface at $S_{\text {nloc1 }}$ depicted in Fig. 3(a). 


\section{E. Magnetic-field dependence of local and nonlocal signals}

The response of $V_{\text {loc }}$ to an external magnetic field in Fig. 5(a) shows a typical weak-antilocalization (WAL) behavior, which has been extensively studied previously $[16,18,19,21,23,55-57]$. In contrast to $V_{\text {loc }}$, however, $V_{\text {nloc1,2 }}$ shows both positive and negative MR, depending on $V_{\mathrm{bg}} . V_{\mathrm{nloc} 1,2}$ is affected by two factors: the change in the total current flowing through $S_{\text {nloc1 }}$ shown in Fig. 3(a) and the change in the resistance of $R_{\text {top }}$. With an increasing magnetic field, $R_{\text {top }}$ also increases according to the WAL effect. If the current profile is not modified, then $V_{\text {nloc1,2 }}$ would increase with the magnetic field. However, because $R_{\text {bot }}$ also increases along with $R_{\text {top }}$, with increasing $B$, $V_{\text {nloc1,2 }}$ depends on the consequent redistribution of current with $V_{\mathrm{bg}}$, which is determined by the variation in the set of $\left(\sigma_{\text {bulk }}, G_{\square}^{\text {top }}, G_{\square}^{\text {bot }}\right)$. By matching the observed results in Figs. 5(a) -5 (c) to the numerical simulation, we find that the complex magnetic-field dependence of $V_{\text {nloc1,2 }}$ in Figs. 5(b) and 5(c) could also be attributed to the combined WAL effect of the top and bottom surfaces with different zero-field conductance [see Figs. 5(d) and 5(e) for the magnetoconductance correction, $\Delta G_{\square}=G_{\square}(B)-G_{\square}(0)$, of the two surfaces].

Because the TSS belongs to the symplectic class, i.e., $\tau_{e}$, $\tau_{\text {so }} \ll \tau_{\phi}$ with $1 / \tau_{s}=0\left(\tau_{e}\right.$ : elastic scattering time; $\tau_{\mathrm{so}}$ : spin-orbit scattering time; $\tau_{\phi}$ : phase relaxation time; $\tau_{s}$ : spin scattering time), the magnetoconductance correction of the TSS is expressed as

$$
\Delta G_{\square}=\alpha \frac{e^{2}}{2 \pi^{2} \hbar}\left[\psi\left(\frac{1}{2}+\frac{\hbar}{4 e l_{\phi}^{2} B}\right)-\ln \left(\frac{\hbar}{4 e l_{\phi}^{2} B}\right)\right],
$$
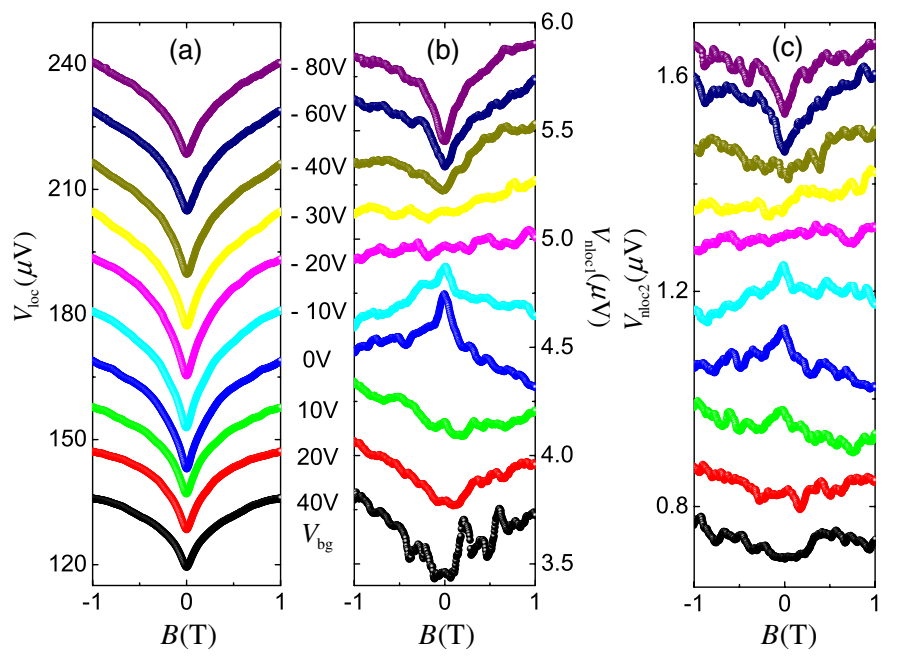

where $\psi$ is the digamma function, $e$ is the electron charge, $\hbar$ is Planck's constant divided by $2 \pi$, and $l_{\phi}$ is the phase relaxation length [58]. The theoretical value of $\alpha$ is -0.5 for each two-dimensional (2D) conducting channel for the symplectic class. The solid lines, in Figs. 5(d) and 5(e), represent the best fits to Eq. (1), in good agreement with WAL behavior. Figures 5(f) and 5(g) show the $V_{\mathrm{bg}}$ dependence of $\alpha$ and $l_{\phi}$, obtained from the best fits in Figs. 5(d) and 5(e). The value of $\alpha$ on the bottom surface (circles) is close to -0.5 , which agrees well with the prediction for a single conducting channel. The relatively large deviation of $\alpha$ from -0.5 near the Dirac point on the bottom surface can be understood by the presence of spatially nonuniform carrier density and carrier types ( $e-h$ puddles), which have been observed on the surface of a TI [59], similar to graphene [60].

In contrast to the bottom surface, the value of $\alpha$ on the top surface (squares) is close to -1 for all $V_{\mathrm{bg}}$. At the surface of a TI, a 2D electron gas (2DEG) is formed in a quantum well set up by band bending. The depth and magnitude of the band bending strongly depend on the surface impurity, carrier and impurity density, temperature, and external potential $[61,62]$. In particular, the 2DEG that forms near the surface of a TI exhibits a large Rashba spin splitting due to strong spin-orbit coupling [8,43,44,63,64]. Because the Rashba spin-split band also has a spinmomentum-locked helical spin texture, it exhibits a WAL effect with $-1<\alpha<0$, depending on the magnitude of spin splitting or the interband scattering strength $[45,58,65,66]$. Therefore, on the top surface, not only the TSS but also the Rashba-split 2DEG contributes to the WAL effect, giving rise to the total value of $\alpha$ close to -1 . It is believed that the difference in the values of $\alpha$ on
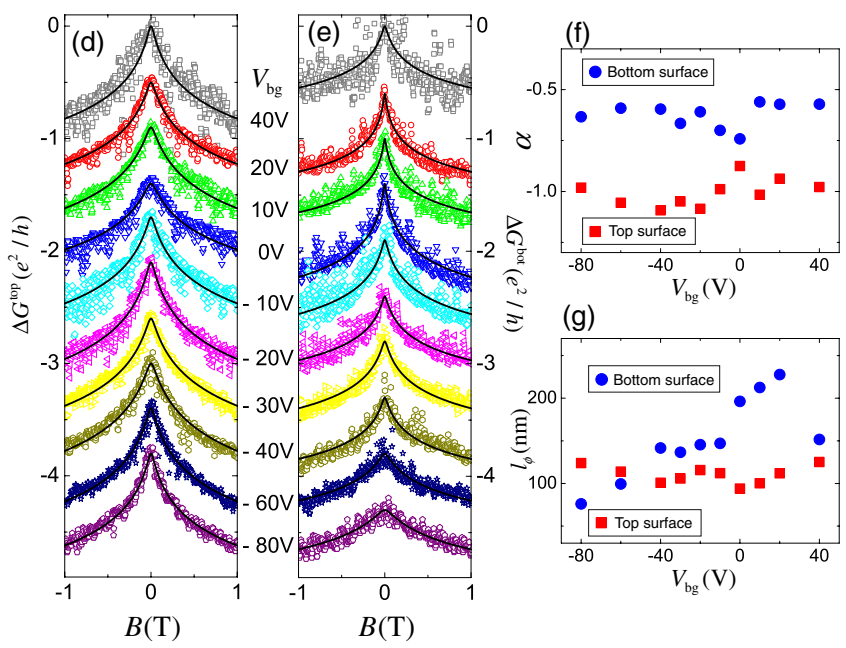

(g)

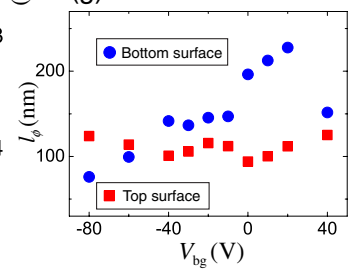

FIG. 5. $\quad V_{\mathrm{bg}}$ dependence of (a) $V_{\text {loc }}$ vs $B$, (b) $V_{\text {nloc1 }}$ vs $B$, and (c) $V_{\text {nloc2 }}$ vs $B$. Each curve is shifted vertically for clarity. The universal conductance fluctuations are conspicuous in $V_{\text {nloc1,2 }}$. (d),(e) Simulation results of $\Delta G_{\square}^{\text {top }}=G_{\square}^{\text {top }}(B)-G_{\square}^{\text {top }}(B=0)$ and $\Delta G_{\square}^{\text {bot }}=$ $G_{\square}^{\text {bot }}(B)-G_{\square}^{\text {bot }}(B=0)$ for a given $V_{\mathrm{bg}}$. The solid lines in (d) and (e) are the best fits to Eq. (1). Each set of data, together with the fitting curve, is shifted vertically for clarity. (f),(g) $V_{\mathrm{bg}}$ dependence of $\alpha$ and $l_{\phi}$ obtained from the best fits to Eq. (1). Squares (circles) correspond to the top (bottom) surface. 
the top and bottom surfaces is caused by doping of the top surface, introduced during electrode patterning and subsequent processes. This result is consistent with our previous observation in BSTS [45]. In Fig. 5(g), $l_{\phi}$ on the top surface is fairly insensitive to $V_{\mathrm{bg}}$, in contrast to $l_{\phi}$ on the bottom surface, which follows the sensitivity of $R_{\square}^{\text {top }}$ and $R_{\square}^{\text {bot }}$ on $V_{\mathrm{bg}}$, as shown in Fig. 3(d). The well-behaving magnetoconductance on the top and bottom surfaces, with highly relevant WAL parameters, extracted out of seemingly contradicting $V_{\mathrm{loc}}(B)$ and $V_{\mathrm{nloc} 1,2}(B)$ on the top surface, conclusively justifies our analysis scheme for the surface conductance in our BSTS TI crystal flakes.

\section{DISCUSSION}

Most of the transport measurements on TI thin films or flakes have been carried out using electrodes arranged on the top surface. The results shown in our study point out that, if the conduction in a 3D TI becomes dominated by the surface conduction, the MR obtained from the top surface should be closely analyzed to extract the correct conduction parameters. The magnetic-field-dependent oscillation of $V_{\text {loc }}$ can be caused not only by the MR oscillation of the top surface but also by the field-dependent oscillation of $I_{\text {top }}$ that is affected by the MR oscillation at the bottom surface. This can lead to multiple-field periodicity in the fast Fourier transform of $R$ vs $\frac{1}{B}$. In fact, this multiple-field periodicity in Shubnikov-de Haas oscillations has been reported previously [67-69]. However, even if the field periodicity corresponding to the top and bottom surfaces is resolved, one still cannot extract various valuable physical parameters, such as the cyclotron mass, mean-free time, and mobility, without precise determination of $\Delta R_{\text {top }}$ and $\Delta R_{\text {bot }}$. To that end, $V_{\text {nloc } 1,2}$, as well as $V_{\text {loc }}$, should be measured using a measurement configuration similar to the one used in this study. The above argument is valid even when the current-biasing electrodes are arranged at the two ends of an elongated TI crystal in contact with its sides, as long as the top and bottom surfaces have substantially different sheet conductances or magnetoconductances.

In transport measurements, a conductance estimation using $I_{\text {bias }} / V_{\text {loc }}$ is valid only when all of the injected bias current $\left(I_{\text {bias }}\right)$ flows locally and uniformly across the two voltage probes. In the presence of both multiple conducting channels and subsequent distributed current flow on the surface of a 3D TI, the conductance cannot simply be estimated from the relation $I_{\text {bias }} / V_{\text {loc }}$. This issue of the distributed current flow becomes more serious when the surface conduction predominates over the bulk conduction. In our previous studies [45], which included only local measurements on a BSTS flake, grown under conditions and thicknesses similar to the ones in this study, we obtained around $6 \%$ of bulk conductance at $4.2 \mathrm{~K}$. Comparing this with $\sigma_{\text {bulk }}$ obtained at $4.2 \mathrm{~K}$ in this study, we believe that the previous estimation was highly overestimated.

\section{CONCLUSION}

In conclusion, we report on the investigation of the distributed current flow over the entire surface of a 3D TI, determined by measuring both $V_{\text {loc }}$ and $V_{\text {nloc1,2 }}$ concurrently. With the topologically robust conducting state on the surface of a TI, the current flow is bound to be distributed all over the surface of a TI, which makes the estimation of the surface conductance based on the local potential measurements readily erroneous. We showed that the surface conductance of the top and bottom surfaces can be estimated with high precision by matching both the local and nonlocal transport measurements on the top surface to the numerical simulation, based on the finite-element method. The backgate-voltage, temperature, and $B$-field dependencies of the conductance on the top and bottom surfaces revealed the high physical relevance. This study offers a highly reliable means of accurately determining the surface conductance of a TSS on a 3D TI, which enables the accurate characterization of the topological conducting state existing on the surface of a TI.

\section{ACKNOWLEDGMENTS}

We would like to thank Stefan Kettemann for valuable discussions. This work was supported by the National Research Foundation (NRF), through the SRC Center for Topological Matter (Grant No. 2011-0030046 for H.-J. L. and J.S.K.), the GFR Center for Advanced Soft Electronics (Grant No. 2011-0031640 for H.-J. L.), and the Mid-Career Researcher Program (Grant No. 2012013838 for J. S. K.).

\section{APPENDIX A: ROBUST SURFACE CONDUCTION IN SIDES OF BSTS SINGLE CRYSTAL}

Figure 6 shows magnified SEM images for different sides of the $\mathrm{Bi}_{1.5} \mathrm{Sb}_{0.5} \mathrm{Te}_{1.7} \mathrm{Se}_{1.3}$ (BSTS) flake used in this study. Because the BSTS is a layered compound, as described in the main text, the side surfaces are rugged or rough when a crystal is exfoliated into a flake. In ordinary band insulators, surface-conducting states that

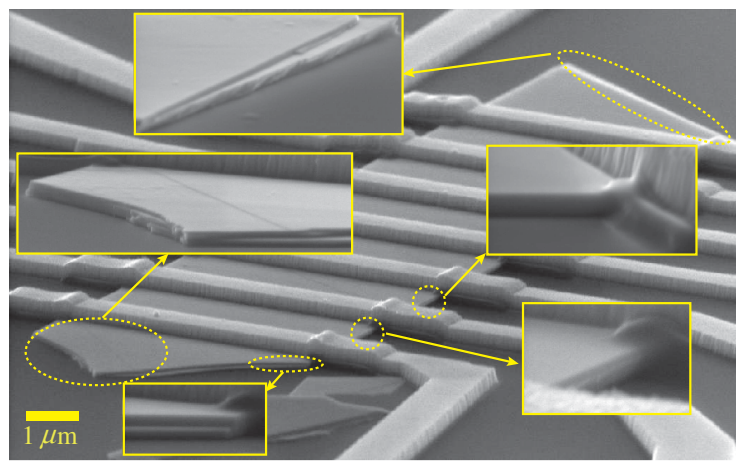

FIG. 6. SEM image of side surfaces of a BSTS flake. Yellow boxes are magnified images for each side part of a BSTS flake. 
may exist in side surfaces cannot be robust. However, in a three-dimensional topological insulator (TI), a gapless boundary conducting state should exist on any surface because of topological protection. Our observation of large nonlocal potential differences in our TI flake is a direct consequence of the existence of this robust gapless surfaceconducting state on the BSTS TI material.

\section{APPENDIX B: ESTIMATING BULK CONDUCTIVITY OF TI}

The value of the base-temperature $(4.2 \mathrm{~K})$ bulk conductivity we obtained in this study turned out to be a few tens of times smaller than the values reported previously for thick BSTS single crystals $[25,38,48]$. In the main text, we stated that $\sigma_{\text {bulk }}$ in the previous studies could have been overestimated because of the coexistence of metallic surface-conducting channels and thermally activated bulk channels. Here, we provide more discussions on that argument. In an ordinary semiconductor or a metal, the bulk conductivity is estimated from the relation

$$
\sigma_{\text {bulk }}^{\exp }=\frac{I_{\text {in }}}{V_{\text {loc }}} \frac{L}{t W},
$$

where $I_{\text {in }}$ is the bias current, $L$ is the spacing between two voltage probes, $V_{\text {loc }}$ is the observed potential difference, and $t$ and $W$ are the thickness and width of a sample, respectively. In fact, $\sigma_{\text {bulk }}^{\text {exp }}$, obtained from Eq. (B1), is the value averaged over all local values of the bulk conductivity, $\sigma_{\text {bulk }}$. Therefore, estimating $\sigma_{\text {bulk }}$ of a material using $\sigma_{\text {bulk }}^{\text {exp }}$ is valid only when the local fluctuation of $\sigma_{\text {bulk }}$ or relative difference of $\sigma_{\text {bulk }}$ between the surface and the bulk is sufficiently small. Estimating $\sigma_{\text {bulk }}$ using relation (B1) is valid for most materials. However, if multiple conducting channels are present and combined in parallel as in 3D TIs, the validity of relation (B1) is no longer guaranteed.

Conducting channels in a bulk TI can be divided into bulk and surface contributions. Separating the latter, the apparent value of bulk conductivity in relation (B1) is modified as

$$
\sigma_{\text {bulk }}^{\exp }=\sigma_{\text {bulk }}+\frac{G_{2 D}}{t},
$$

where $G_{2 D}$ is the surface sheet conductance and $t$ is the thickness of the sample. If $\sigma_{\text {bulk }}$ is sufficiently large so as to neglect the surface contribution, $\sigma_{\text {bulk }}^{\exp } \approx \sigma_{\text {bulk }}$. This corresponds to ordinary semiconductors and conductors, with relatively low resistivity of about $\mathrm{m} \Omega \mathrm{cm}$.

For a BSTS bulk crystal of thickness $t=100 \mu \mathrm{m}$, with the Fermi level $\left(E_{F}\right)$ of the surface state located around the middle between the bottom of the conduction band and the Dirac point, the carrier density is about $n_{s} \approx 2 \times 10^{12} \mathrm{~cm}^{-2}$ (note that the maximum carrier density, without touching the conduction band, is about $n_{s} \approx 5 \times 10^{12} \mathrm{~cm}^{-2}$ ). Then, assuming the carrier mobility to be around $1000 \mathrm{~cm}^{2} /(\mathrm{V} \cdot \mathrm{s})$ (these assumptions are reasonable, and a variation of the values does not affect the result of our discussion), along with the relation $\sigma=n e \mu$, the sheet conductance of each surface becomes around $300 \mu \mathrm{S}$ (corresponds to about $8.3 e^{2} / h$ ). For the top and bottom surfaces together, $G_{2 D} \approx 600 \mu \mathrm{S}$. Thus, Eq. (B2) leads to

$$
\sigma_{\text {bulk }}^{\exp } \sim \sigma_{\text {bulk }}+6 \mathrm{~S} / \mathrm{m} \text {. }
$$

If $\sigma_{\text {bulk }}^{\exp }$ is comparable to around $10 \mathrm{~S} / \mathrm{m}$, as in previous reports on BSTS [25,35,38,49-53], $\sigma_{\text {bulk }}^{\exp }$ no longer represents the true value, $\sigma_{\text {bulk }}$. Thus, in the presence of the separate surface-conducting channels in a TI with appreciable surface conductance, the apparent value of the bulk conductivity, $\sigma_{\text {bulk }}^{\exp }$, can be significantly larger than the true value, $\sigma_{\text {bulk }}$.

In addition to the topological surface-conducting channels, the conduction by the $2 \mathrm{D}$ electron gas formed in the quantum well due to the surface band bending also enhances the surface conductance $G_{2 D}$. The surface band bending is a common feature at the surface of a semiconductor, especially in a narrow-band-gap semiconductor like TIs. Thus, in a thick bulk TI crystal, multiple surfaceconducting channels, other than the bulk-conducting channels, contribute to $G_{2 D}$ and enhance the apparent bulk conductivity, $\sigma_{\text {bulk }}^{\exp }$, in Eq. (B1). In thin flakes, as in our samples, the dominant surface-conducting channels, connected in parallel to the bulk channels, make the estimation of the bulk conductivity impossible. In this study, by resorting to measurements of the nonlocal potential difference and the numerical simulation, we were able to set the upper limit value of $\sigma_{\text {bulk }}$ to about $0.6 \mathrm{~S} / \mathrm{m}$ at $4.2 \mathrm{~K}$. For the reasons discussed above, we suspect that the values from previous studies were overestimated.

\section{APPENDIX C: DETAILS AND VALIDITY OF NUMERICAL SIMULATION METHOD}

We used the commercial software package (COMSOL) [36] to calculate the current distribution on the surface of a 3D TI, based on the finite-element method. Figure 7(a) shows the top view of our simulation model. For the numerical simulation, we used the actual sample dimensions (see Table I), which were obtained from the atomic force microscopy and SEM. To exclude the unintended electrical contact at the sides of our BSTS flake, we covered the side edges underneath the Ti/Au electrodes with crosslinked PMMA $950 \mathrm{~K} \mathrm{C4}$ as an insulating layer. All the edge-to-edge spacings between two neighboring electrodes are identically $1.9 \mu \mathrm{m}$. Figure 7(b) is the cross section of the simulation model, and Fig. 7(c) shows a magnified view. We set the thickness of the surface-conducting channel $\left(t_{s}\right)$ to be $5 \mathrm{~nm}$ in the simulation [see Fig. 7(c)]. In fact, the spatial distribution of $|\psi|^{2}[\psi$ is the wave function of the TSS in a TI] is at most 1-2 quintuple layers 


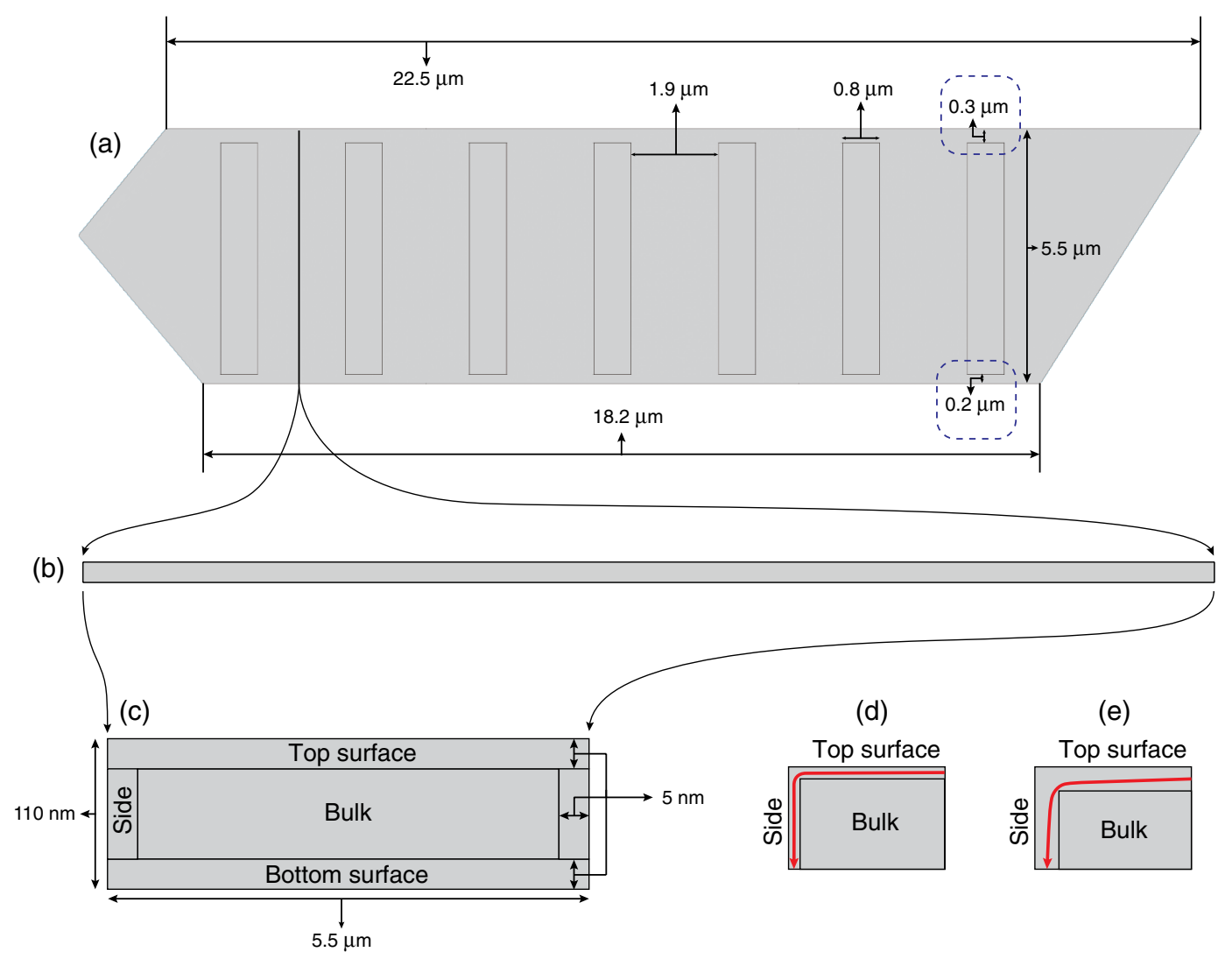

FIG. 7. (a) Schematic top view of the BSTS flake. (b) The cross section of the BSTS flake with the actual aspect ratio. The surface channel regions cannot be shown in this schematic figure because of the small surface thickness (about 5 nm) compared to the thickness of the flake (about $110 \mathrm{~nm}$ ). (c) An exaggerated view of the cross section of the BSTS flake corresponding to the region shown in (b). (d),(e) Influence of the surface thickness on the current path near the edge.

(QL), which corresponds to 1-2 $\mathrm{nm}$, depending on the van der Waals gap, i.e., the spacing between adjacent QLs [70-72]. In addition to the TSS, ordinary conducting channels of 2DEG form on the surface of a TI. The range of $|\psi|^{2}$ of electrons in a 2DEG can be up to nm, depending on the position of $E_{F}$ and the impurity density [8,43,44]. Because carriers in both the TSS and the 2DEG have a common 2D nature, the accurate value of $t_{s}$ in numerical simulations is not crucial. The sheet conductances of the top and bottom surfaces $\left(G_{\square}^{\text {top }}\right.$ and $\left.G_{\square}^{\text {bot }}\right)$ matter the most.

In fact, there is little difference between the simulation models for different $t_{s}$. Figures 7(d) and 7(e) show the different simulation results for two models with different $t_{s}$. If $t_{s}$ increases [from Fig. 7(d) to Fig. 7(e)], the electrical current takes a shorter path near the edge region [see Fig. 7(e)]. Then, the current density profile should change according to the simulation results. However, as shown in Fig. 7(b), because the aspect ratio of the cross section in our flake is very large, $t_{s}$ is hardly perceived; then, the difference between simulation models with different $t_{s}$ is almost negligible.

Figure 8 shows the simulation models for different $t_{s}$ $(20 \mathrm{~nm}, 10 \mathrm{~nm}, 5 \mathrm{~nm}, 4 \mathrm{~nm}, 3 \mathrm{~nm})$ with the same $G_{\square}^{\text {top }}$ and $G_{\square}^{\text {bot }}$. The effect of $t_{s}$ variation on the simulation result of
$V_{\text {loc }}$ and $V_{\text {nloc1 }}$ is very small. Even the small difference between $t_{s}=20 \mathrm{~nm}$ and $t_{s}=10 \mathrm{~nm}$ almost disappears as $t_{s}$ decreases below $5 \mathrm{~nm}$ [see the inset in Fig. 8(b)]. Therefore, we conclude that the simulation results for the model of $t_{s}=1-2 \mathrm{~nm}$ should be almost the same as our model of $t_{s}=5 \mathrm{~nm}$. Lastly, in the numerical simulation, we set $G_{\square}^{\text {side }}$ equal to $G_{\square}^{\text {top }}$. Because the area of the side surface is very small compared to that of the top and bottom surfaces, the effect of a small ambiguity in the value
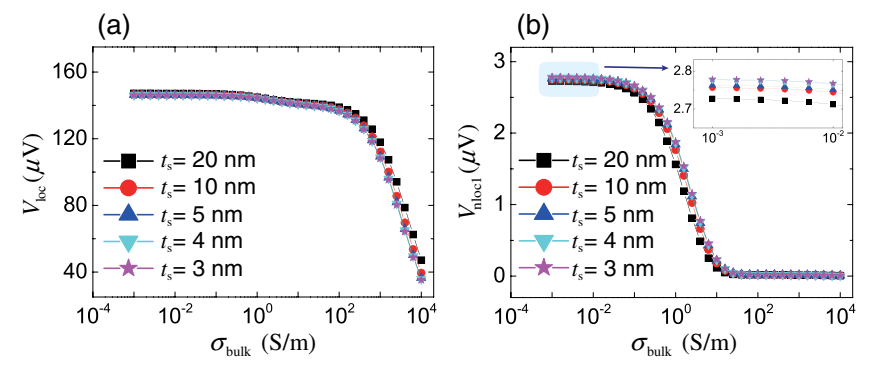

FIG. 8. Surface-channel-thickness dependence of the simulation results for (a) $V_{\mathrm{loc}}$ and (b) $V_{\text {nloc1 }}$, corresponding to $\left(G_{\square}^{\text {top }}, G_{\square}^{\text {bot }}\right)=(6.0,2.5)\left(e^{2} / h\right)$. The inset of (b) represents the magnified view of the marked region. 
of $G_{\square}^{\text {side }}$ hardly affects the results of a set of ( $\sigma_{\text {bulk }}, G_{\square}^{\text {top }}, G_{\square}^{\text {bot }}$ ) unless the $G_{\square}^{\text {side }}$ is significantly smaller than $G_{\square}^{\text {top }}$.

\section{APPENDIX D: $T$ DEPENDENCE OF LOCAL AND NONLOCAL SIGNALS}

The $T$ dependence of $V_{\text {nloc1 }}$ and $V_{\text {nloc2 }}$ in Fig. 1(c) in the main text is in discordance with that of $V_{\text {loc }}$. $V_{\text {loc }}$ continues to decrease below around $80 \mathrm{~K}$, while $V_{\text {nloc1 }}$ and $V_{\text {nloc2 }}$ slightly increase and saturate, respectively. This seems to be somewhat inconsistent with the behavior of metallic surfaces. Figures 9(a) and 9(b) show the $T$ dependence of $G_{\square}^{\text {top }}$ and $G_{\square}^{\text {bot }}$ for $V_{\mathrm{bg}}=0 \mathrm{~V}$, respectively [the inset of Fig. 9(b) shows $\sigma_{\text {bulk }}$ vs $T$ ], which were obtained from the values of $V_{\text {loc }}, V_{\text {nloc1 }}$, and $V_{\text {nloc2 }}$ for $T$ below $70 \mathrm{~K}$ (where the simulation results were largely insensitive to the variation of $\sigma_{\text {bulk }}$ ), in the same manner as used to obtain the temperature dependence of the variables in Fig. 3(d).

As mentioned in Appendix C, we set the $t_{s}$ to be $5 \mathrm{~nm}$, providing the justification. However, as the temperature increases, this simple model is no longer applicable. At high temperatures (in our sample, $T>70 \mathrm{~K}$ ), the boundary between the bulk-conducting region and the surfaceconducting layer becomes obscure as thermally excited bulk carriers increase and the carrier-confining surface potential well becomes broadened. Consequently, our simulation model in Fig. 7 can be applied only for a sufficiently low temperature region, where the boundary between surface and bulk-conducting channels can be well defined. We found that our simulation failed to provide solutions for the observed values of $V_{\text {loc }}, V_{\text {nloc1 }}$, and $V_{\text {nloc2 }}$, for any combination set of ( $\left.\sigma_{\text {bulk }}, G_{\square}^{\text {top }}, G_{\square}^{\text {bot }}\right)$, for temperatures above $70 \mathrm{~K}$. Thus, here, we provide only a qualitative explanation of the observed temperature dependence of $V_{\text {loc }}, V_{\text {nloc1 } 1}$, and $V_{\text {nloc2 }}$ in Fig. 1(c).

The total local current between the source and drain constitutes most of the bias current and thus does not vary significantly over the entire temperature range of
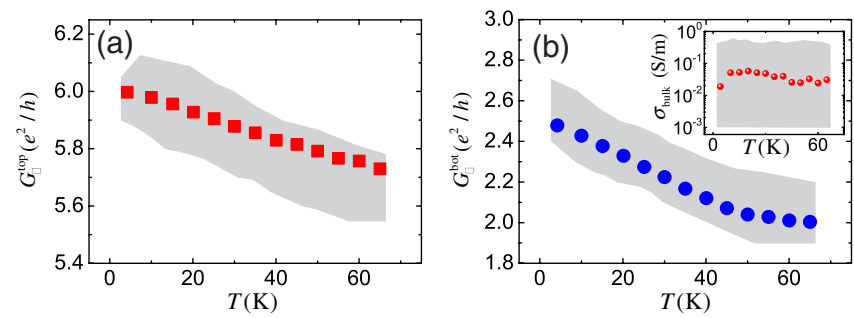

FIG. 9. Simulation results of $G_{\square}^{\text {top }}$ and $G_{\square}^{\text {bot }}$, leading to the matching condition for the experimental data of $V_{\text {loc }}, V_{\text {nloc } 1}$, and $V_{\text {nloc2 }}$ simultaneously for temperatures in the range $T<70 \mathrm{~K}$. The inset in (b) shows the corresponding temperature variation of $\sigma_{\text {bulk }}$. Grey shaded regions indicate the range of the variables giving rise to the observed data (with fluctuations) within an uncertainty of $\left|\Delta V_{\text {loc }}\right| \leq 1 \mu \mathrm{V}, \quad\left|\Delta V_{\text {nloc } 1}\right| \leq 0.3 \mu \mathrm{V}$, and $\left|\Delta V_{\text {nloc } 2}\right| \leq 0.05 \mu \mathrm{V}$. The upper limit of $\sigma_{\text {bulk }}$ is $\sim 0.6 \mathrm{~S} / \mathrm{m}$. Symbols indicate the weighted average values. measurements. Here, the local current through the bulk, which dominates the bias current at high temperatures, is simply redistributed with decreasing $T$ into top and bottom surfaces between the source and drain. Thus, $V_{\text {loc }}(T)$ reflects the $T$ dependence of the effective sample conductance via the bulk and surface-conducting channels connected in parallel between the source and drain. In the high- $T$ region, where the bulk conductance dominates the effective resistance, $V_{\text {loc }}$ increases quickly with decreasing $T$. However, in the low-temperature range, where the effective resistance between the source and drain is mostly governed by the surface conductance with metallic $T$ dependence, $V_{\text {loc }}$ decreases with decreasing $T$. The broad crossover of high-to-low $T$ behavior in $V_{\text {loc }}$ took place at $80-100 \mathrm{~K}$ in our flake. In contrast, the $T$ dependence of $V_{\text {nloc } 1,2}$, which is determined by $I_{\text {nloc } 1,2}(T) \times 1 / G_{\square}^{\text {top }}(T)$, is more complicated. Here, the minute nonlocal current $I_{\text {nloc } 1,2}(T)$ arises from part of the bias current leaking out of the local region, as the current flow through the bulk is blocked in the local region. The $T$ dependence of $I_{\text {nloc } 1,2}(T)$ is determined by the combined effect from $\sigma_{\text {bulk }}(T), G_{\square}^{\text {top }}(T)$, and $G_{\square}^{\text {bot }}(T)$. At sufficiently low $T$, below $80-100 \mathrm{~K}$, however, as most of the bias current is taken over by the surface current, the rate of increase of $I_{\text {nloc1,2 }}$ with decreasing $T$ drops sharply. Combining this with a slow metallic increase of $G_{\square}^{\text {top }}(T)$ gives rise to almost temperature-independent $V_{\text {nloc1,2 }}(T)$ below $80-100 \mathrm{~K}$ as in Fig. 1(c). But, with decreasing $T$ above $100 \mathrm{~K}$, a combination of a faster increase in $I_{\text {nloc1,2 }}(T)$ and a metallic increase in $G_{\square}^{\text {top }}$ leads to a maximum in $V_{\text {nloc1,2 }}$ in the temperature range of $100<T<300 \mathrm{~K}$. Detailed temperature dependencies of $V_{\text {loc }}, V_{\text {nloc1 }}$, and $V_{\text {nloc2 }}$ in Fig. 1(c) resulted from the combined effect among different transport parameters.

[1] H. Zhang, C.-X. Liu, X.-L. Qi, X. Dai, Z. Fang, and S.-C. Zhang, Topological Insulators in $\mathrm{Bi}_{2} \mathrm{Se}_{3}, \mathrm{Bi}_{2} \mathrm{Te}_{3}$ and $\mathrm{Sb}_{2} \mathrm{Te}_{3}$ with a Single Dirac Cone on the Surface, Nat. Phys. 5, 438 (2009).

[2] D. Hsieh, D. Qian, L. Wray, Y. Xia, Y. S. Hor, R. J. Cava, and M.Z. Hasan, A Topological Dirac Insulator in a Quantum Spin Hall Phase, Nature (London) 452, 970 (2008).

[3] D. Hsieh, Y. Xia, L. Wray, D. Qian, A. Pal, J. H. Dil, J. Osterwalder, F. Meier, G. Bihlmayer, C. L. Kane, Y. S. Hor, R. J. Cava, and M.Z. Hasan, Observation of Unconventional Quantum Spin Textures in Topological Insulators, Science 323, 919 (2009).

[4] Y. L. Chen, J. G. Analytis, J.-H. Chu, Z. K. Liu, S.-K. Mo, X. L. Qi, H. J. Zhang, D. H. Lu, X. Dai, Z. Fang, S. C. Zhang, I. R. Fisher, Z. Hussain, and Z.-X. Shen, Experimental Realization of a Three-Dimensional Topological Insulator, $\mathrm{Bi}_{2} \mathrm{Te}_{3}$, Science 325, 178 (2009).

[5] D. Hsieh, Y. Xia, D. Qian, L. Wray, J. H. Dil, F. Meier, J. Osterwalder, L. Patthey, J. G. Checkelsky, N. P. Ong, 
A. V. Fedorov, H. Lin, A. Bansil, D. Grauer, Y. S. Hor, R. J. Cava, and M. Z. Hasan, A Tunable Topological Insulator in the Spin Helical Dirac Transport Regime, Nature (London) 460, 1101 (2009).

[6] L. Fu, C. L. Kane, and E. J. Mele, Topological Insulators in Three Dimensions, Phys. Rev. Lett. 98, 106803 (2007).

[7] J.E. Moore and L. Balents, Topological Invariants of Time-Reversal-Invariant Band Structures, Phys. Rev. B 75, 121306 (2007).

[8] M. S. Bahramy, P. D. C. King, A. de la Torre, J. Chang, M. Shi, L. Patthey, G. Balakrishnan, P. Hofmann, R. Arita, N. Nagaosa, and F. Baumberger, Emergent Quantum Confinement at Topological Insulator Surfaces, Nat. Commun. 3, 1159 (2012).

[9] K. Miyamoto, A. Kimura, T. Okuda, H. Miyahara, K. Kuroda, H. Namatame, M. Taniguchi, S. V. Eremeev, T. V. Menshchikova, E. V. Chulkov, K. A. Kokh, and O. E. Tereshchenko, Topological Surface States with Persistent High Spin Polarization Across the Dirac Point in $\mathrm{Bi}_{2} \mathrm{Te}_{2} \mathrm{Se}$ and $\mathrm{Bi}_{2} \mathrm{Se}_{2}$ Te, Phys. Rev. Lett. 109, 166802 (2012).

[10] X.-L. Qi, R. Li, J. Zang, and S.-C. Zhang, Inducing a Magnetic Monopole with Topological Surface States, Science 323, 1184 (2009).

[11] B. Seradjeh, J. E. Moore, and M. Franz, Exciton Condensation and Charge Fractionalization in a Topological Insulator Film, Phys. Rev. Lett. 103, 066402 (2009).

[12] L. Fu and C. L. Kane, Superconducting Proximity Effect and Majorana Fermions at the Surface of a Topological Insulator, Phys. Rev. Lett. 100, 096407 (2008).

[13] R. Yu, W. Zhang, H.-J. Zhang, S.-C. Zhang, X. Dai, and Z. Fang, Quantized Anomalous Hall Effect in Magnetic Topological Insulators, Science 329, 61 (2010).

[14] J. G. Analytis, J.-H. Chu, Y. Chen, F. Corredor, R. D. McDonald, Z. X. Shen, and I. R. Fisher, Bulk Fermi Surface Coexistence with Dirac Surface State in $\mathrm{Bi}_{2} \mathrm{Se}_{3}$ : A Comparison of Photoemission and Shubnikov-de Haas Measurements, Phys. Rev. B 81, 205407 (2010).

[15] J. G. Analytis, R. D. McDonald, S. C. Riggs, J.-H. Chu, G. S. Boebinger, and I. R. Fisher, Two-Dimensional Surface State in the Quantum Limit of a Topological Insulator, Nat. Phys. 6, 960 (2010).

[16] J. Chen, H. J. Qin, F. Yang, J. Liu, T. Guan, F. M. Qu, G. H. Zhang, J. R. Shi, X. C. Xie, C. L. Yang, K. H. Wu, Y. Q. Li, and L. Lu, Gate-Voltage Control of Chemical Potential and Weak Antilocalization in $\mathrm{Bi}_{2} \mathrm{Se}_{3}$, Phys. Rev. Lett. 105, 176602 (2010).

[17] K. Eto, Z. Ren, A. A. Taskin, K. Segawa, and Y. Ando, Angular-Dependent Oscillations of the Magnetoresistance in $\mathrm{Bi}_{2} \mathrm{Se}_{3}$ Due to the Three-Dimensional Bulk Fermi Surface, Phys. Rev. B 81, 195309 (2010).

[18] J. G. Checkelsky, Y. S. Hor, R. J. Cava, and N. P. Ong, Bulk Band Gap and Surface State Conduction Observed in Voltage-Tuned Crystals of the Topological Insulator $\mathrm{Bi}_{2} \mathrm{Se}_{3}$, Phys. Rev. Lett. 106, 196801 (2011).

[19] J. Chen, X. Y. He, K. H. Wu, Z. Q. Ji, L. Lu, J. R. Shi, J. H. Smet, and Y. Q. Li, Tunable Surface Conductivity in $\mathrm{Bi}_{2} \mathrm{Se}_{3}$ Revealed in Diffusive Electron Transport, Phys. Rev. B 83, 241304 (2011).

[20] D. Kong, Y. Chen, J. J. Cha, Q. Zhang, J. G. Analytis, K. Lai, Z. Liu, S. S. Hong, K. J. Koski, S.-K. Mo, Z. Hussain,
I. R. Fisher, Z.-X. Shen, and Y. Cui, Ambipolar Field Effect in the Ternary Topological Insulator $\left(\mathrm{Bi}_{x} \mathrm{Sb}_{1-x}\right)_{2} \mathrm{Te}_{3}$ by Composition Tuning, Nat. Nanotechnol. 6, 705 (2011).

[21] H. Steinberg, J.-B. Laloë, V. Fatemi, J. S. Moodera, and P. Jarillo-Herrero, Electrically Tunable Surface-to-Bulk Coherent Coupling in Topological Insulator Thin Films, Phys. Rev. B 84, 233101 (2011).

[22] H. Cao, J. Tian, I. Miotkowski, T. Shen, J. Hu, S. Qiao, and Y. P. Chen, Quantized Hall Effect and Shubnikov-de Haas Oscillations in Highly Doped $\mathrm{Bi}_{2} \mathrm{Se}_{3}$ : Evidence for Layered Transport of Bulk Carriers, Phys. Rev. Lett. 108, 216803 (2012).

[23] S. S. Hong, J. J. Cha, D. Kong, and Y. Cui, Ultra-Low Carrier Concentration and Surface-Dominant Transport in Antimony-Doped $\mathrm{Bi}_{2} \mathrm{Se}_{3}$ Topological Insulator Nanoribbons, Nat. Commun. 3, 757 (2012).

[24] F. Xiu, L. He, Y. Wang, L. Cheng, L.-T. Chang, M. Lang, G. Huang, X. Kou, Y. Zhou, X. Jiang, Z. Chen, J. Zou, A. Shailos, and K. L. Wang, Manipulating Surface States in Topological Insulator Nanoribbons, Nat. Nanotechnol. 6, 216 (2011).

[25] A. A. Taskin, Z. Ren, S. Sasaki, K. Segawa, and Y. Ando, Observation of Dirac Holes and Electrons in a Topological Insulator, Phys. Rev. Lett. 107, 016801 (2011).

[26] J. G. Checkelsky, Y. S. Hor, M. H. Liu, D. X. Qu, R. J. Cava, and N.P. Ong, Quantum Interference in Macroscopic Crystals of Nonmetallic $\mathrm{Bi}_{2} \mathrm{Se}_{3}$, Phys. Rev. Lett. 103, 246601 (2009).

[27] D.-X. Qu, Y. S. Hor, J. Xiong, R. J. Cava, and N. P. Ong, Quantum Oscillations and Hall Anomaly of Surface States in the Topological Insulator $\mathrm{Bi}_{2} \mathrm{Te}_{3}$, Science 329, 821 (2010).

[28] G. Zhang, H. Qin, J. Chen, X. He, L. Lu, Y. Li, and K. Wu, Growth of Topological Insulator $\mathrm{Bi}_{2} \mathrm{Se}_{3}$ Thin Films on $\mathrm{SrTiO}_{3}$ with Large Tunability in Chemical Potential, Adv. Funct. Mater. 21, 2351 (2011).

[29] M. Petrushevsky, E. Lahoud, A. Ron, E. Maniv, I. Diamant, I. Neder, S. Wiedmann, V. K. Guduru, F. Chiappini, U. Zeitler, J. C. Maan, K. Chashka, A. Kanigel, and Y. Dagan, Probing the Surface States in $\mathrm{Bi}_{2} \mathrm{Se}_{3}$ Using the Shubnikovde Haas Effect, Phys. Rev. B 86, 045131 (2012).

[30] B. A. Assaf, T. Cardinal, P. Wei, F. Katmis, J. S. Moodera, and D. Heiman, Linear Magnetoresistance in Topological Insulator Thin Films: Quantum Phase Coherence Effects at High Temperatures, Appl. Phys. Lett. 102, 012102 (2013).

[31] H. Köhler and E. Wöchner, The g-Factor of the Conduction Electrons in $\mathrm{Bi}_{2} \mathrm{Se}_{3}$, Phys. Status Solidi B 67, 665 (1975).

[32] P. Cheng, C. Song, T. Zhang, Y. Zhang, Y. Wang, J.-F. Jia, J. Wang, Y. Wang, B.-F. Zhu, X. Chen, X. Ma, K. He, L. Wang, X. Dai, Z. Fang, X. Xie, X.-L. Qi, C.-X. Liu, S.-C. Zhang, and Q.-K. Xue, Landau Quantization of Topological Surface States in $\mathrm{Bi}_{2} \mathrm{Se}_{3}$, Phys. Rev. Lett. 105, 076801 (2010).

[33] T. Hanaguri, K. Igarashi, M. Kawamura, H. Takagi, and T. Sasagawa, Momentum-Resolved Landau-Level Spectroscopy of Dirac Surface State in $\mathrm{Bi}_{2} \mathrm{Se}_{3}$, Phys. Rev. B 82, 081305 (2010).

[34] Y. Jiang, Y. Wang, M. Chen, Z. Li, C. Song, K. He, L. Wang, X. Chen, X. Ma, and Q.-K. Xue, Landau Quantization and the Thickness Limit of Topological Insulator Thin Films of $\mathrm{Sb}_{2} \mathrm{Te}_{3}$, Phys. Rev. Lett. 108, 016401 (2012). 
[35] J. Xiong, Y. Luo, Y. Khoo, S. Jia, R. J. Cava, and N. P. Ong, High-Field Shubnikov-de Haas Oscillations in the Topological Insulator $\mathrm{Bi}_{2} \mathrm{Te}_{2} \mathrm{Se}$, Phys. Rev. B 86, 045314 (2012).

[36] COMSOL, http://www.comsol.com.

[37] T. Arakane, T. Sato, S. Souma, K. Kosaka, K. Nakayama, M. Komatsu, T. Takahashi, Z. Ren, K. Segawa, and Y. Ando, Tunable Dirac Cone in the Topological Insulator $\mathrm{Bi}_{2-x} \mathrm{Sb}_{x} \mathrm{Te}_{3-y} \mathrm{Se}_{y}$, Nat. Commun. 3, 636 (2012).

[38] Z. Ren, A. A. Taskin, S. Sasaki, K. Segawa, and Y. Ando, Optimizing $\mathrm{Bi}_{2-x} \mathrm{Sb}_{x} \mathrm{Te}_{3-y} \mathrm{Se}_{y}$ Solid Solutions to Approach the Intrinsic Topological Insulator Regime, Phys. Rev. B 84, 165311 (2011).

[39] W. Ko, I. Jeon, H. W. Kim, H. Kwon, S.-J. Kahng, J. Park, J. S. Kim, S. W. Hwang, and H. Suh, Atomic and Electronic Structure of an Alloyed Topological Insulator, $\mathrm{Bi}_{1.5} \mathrm{Sb}_{0.5} \mathrm{Te}_{1.7} \mathrm{Se}_{1.3}$, Sci. Rep. 3, 2656 (2013).

[40] A. Roth, C. Brüne, H. Buhmann, L. W. Molenkamp, J. Maciejko, X.-L. Qi, and S.-C. Zhang, Nonlocal Transport in the Quantum Spin Hall state, Science 325, 294 (2009).

[41] J. Balakrishnan, G. Kok Wai Koon, M. Jaiswal, A. H. Castro Neto, and B. Özyilmaz, Colossal Enhancement of SpinOrbit Coupling in Weakly Hydrogenated Graphene, Nat. Phys. 9, 284 (2013).

[42] G. Mihajlović, J. E. Pearson, M. A. Garcia, S. D. Bader, and A. Hoffmann, Negative Nonlocal Resistance in Mesoscopic Gold Hall Bars: Absence of the Giant Spin Hall Effect, Phys. Rev. Lett. 103, 166601 (2009).

[43] P. D. C. King et al., Large Tunable Rashba Spin Splitting of a Two-Dimensional Electron Gas in $\mathrm{Bi}_{2} \mathrm{Se}_{3}$, Phys. Rev. Lett. 107, 096802 (2011).

[44] H. M. Benia, C. Lin, K. Kern, and C. R. Ast, Reactive Chemical Doping of the $\mathrm{Bi}_{2} \mathrm{Se}_{3}$ Topological Insulator, Phys. Rev. Lett. 107, 177602 (2011).

[45] J. Lee, J. Park, J.-H. Lee, J. S. Kim, and H.-J. Lee, GateTuned Differentiation of Surface-Conducting States in $\mathrm{Bi}_{1.5} \mathrm{Sb}_{0.5} \mathrm{Te}_{1.7} \mathrm{Se}_{1.3}$ Topological-Insulator Thin Crystals, Phys. Rev. B 86, 245321 (2012).

[46] Y.-W. Tan, Y. Zhang, K. Bolotin, Y. Zhao, S. Adam, E. H. Hwang, S. Das Sarma, H. L. Stormer, and P. Kim, Measurement of Scattering Rate and Minimum Conductivity in Graphene, Phys. Rev. Lett. 99, 246803 (2007).

[47] J. Wang, X. Chen, B.-F. Zhu, and S.-C. Zhang, Topological p-n Junction, Phys. Rev. B 85, 235131 (2012).

[48] B. Xia, P. Ren, A. Sulaev, P. Liu, S.-Q. Shen, and L. Wang, Indications of Surface-Dominated Transport in Single Crystalline Nano Flake Devices of Topological Insulator $\mathrm{Bi}_{1.5} \mathrm{Sb}_{0.5} \mathrm{Te}_{1.8} \mathrm{Se}_{1.2}$, Phys. Rev. B 87, 085442 (2013).

[49] J. Xiong, Y. Khoo, S. Jia, R. J. Cava, and N. P. Ong, Tuning the Quantum Oscillations of Surface Dirac Electrons in the Topological Insulator $\mathrm{Bi}_{2} \mathrm{Te}_{2} \mathrm{Se}$ by Liquid Gating, Phys. Rev. B 88, 035128 (2013).

[50] Z. Ren, A. A. Taskin, S. Sasaki, K. Segawa, and Y. Ando, Fermi Level Tuning and a Large Activation Gap Achieved in the Topological Insulator $\mathrm{Bi}_{2} \mathrm{Te}_{2}$ Se by Sn Doping, Phys. Rev. B 85, 155301 (2012).

[51] Z. Ren, A. A. Taskin, S. Sasaki, K. Segawa, and Y. Ando, Large Bulk Resistivity and Surface Quantum Oscillations in the Topological Insulator $\mathrm{Bi}_{2} \mathrm{Te}_{2} \mathrm{Se}$, Phys. Rev. B 82, 241306 (2010).
[52] J. Xiong, A. C. Petersen, D. Qu, Y. S. Hor, R. J. Cava, and N. P. Ong, Quantum Oscillations in a Topological Insulator $\mathrm{Bi}_{2} \mathrm{Te}_{2} \mathrm{Se}$ with Large Bulk Resistivity (6 $\left.\Omega \mathrm{cm}\right)$, Physica (Amsterdam) 44E, 917 (2012).

[53] K. Segawa, Z. Ren, S. Sasaki, T. Tsuda, S. Kuwabata, and Y. Ando, Ambipolar Transport in Bulk Crystals of a Topological Insulator by Gating with Ionic Liquid, Phys. Rev. B 86, 075306 (2012).

[54] There is no supporting report on nonzero off-diagonal components of the conductance tensor.

[55] J. J. Cha, D. Kong, S.-S. Hong, J. G. Analytis, K. Lai, and Y. Cui, Weak Antilocalization in $\mathrm{Bi}_{2}\left(\mathrm{Se}_{x} \mathrm{Te}_{1-x}\right)_{3}$ Nanoribbons and Nanoplates, Nano Lett. 12, 1107 (2012).

[56] A. A. Taskin, S. Sasaki, K. Segawa, and Y. Ando, Manifestation of Topological Protection in Transport Properties of Epitaxial $\mathrm{Bi}_{2} \mathrm{Se}_{3}$ Thin Films, Phys. Rev. Lett. 109, 066803 (2012).

[57] M. Lang, L. He, X. Kou, P. Upadhyaya, Y. Fan, H. Chu, Y. Jiang, J. H. Bardarson, W. Jiang, E. S. Choi, Y. Wang, N.-C. Yeh, J. Moore, and K. L. Wang, Competing Weak Localization and Weak Antilocalization in Ultrathin Topological Insulators, Nano Lett. 13, 48 (2013).

[58] S. Hikami, A. I. Larkin, and Y. Nagaoka, Spin-Orbit Interaction and Magnetoresistance in the Two Dimensional Random System, Prog. Theor. Phys. 63, 707 (1980).

[59] H. Beidenkopf, P. Roushan, J. Seo, L. Gorman, I. Drozdov, Y. S. Hor, R. J. Cava, and A. Yazdani, Spatial Fluctuations of Helical Dirac Fermions on the Surface of Topological Insulators, Nat. Phys. 7, 939 (2011).

[60] J. Martin, N. Akerman, G. Ulbricht, T. Lohmann, J. H. Smet, K. von Klitzing, and A. Yacoby, Observation of Electron-Hole Puddles in Graphene Using a Scanning Single-Electron Transistor, Nat. Phys. 4, 144 (2008).

[61] T. Ando, A. B. Fowler, and F. Stern, Electronic Properties of Two-Dimensional Systems, Rev. Mod. Phys. 54, 437 (1982).

[62] A. B. Fowler, Substrate Bias Effects on Electron Mobility in Silicon Inversion Layers at Low Temperatures, Phys. Rev. Lett. 34, 15 (1975).

[63] Y. A. Bychkov and E. I. Rashba, Properties of a $2 d$ Electron Gas with Lifted Spectral Degeneracy, JETP Lett. 39, 78 (1984).

[64] Y. A. Bychkov and E. I. Rashba, Oscillatory Effects and the Magnetic Susceptibility of Carriers in Inversion Layers, J. Phys. C 17, 6039 (1984).

[65] J. H. Bardarson and J. E. Moore, Quantum Interference and Aharonov-Bohm Oscillations in Topological Insulators, Rep. Prog. Phys. 76, 056501 (2013).

[66] I. Garate and L. Glazman, Weak Localization and Antilocalization in Topological Insulator Thin Films with Coherent Bulk-Surface Coupling, Phys. Rev. B 86, 035422 (2012).

[67] Z. Ren, A. A. Taskin, S. Sasaki, K. Segawa, and Y. Ando, Observations of Two-Dimensional Quantum Oscillations and Ambipolar Transport in the Topological Insulator $\mathrm{Bi}_{2} \mathrm{Se}_{3}$ Achieved by Cd Doping, Phys. Rev. B 84, 075316 (2011).

[68] A. A. Taskin, S. Sasaki, K. Segawa, and Y. Ando, Achieving Surface Quantum Oscillations in Topological Insulator Thin Films of $\mathrm{Bi}_{2} \mathrm{Se}_{3}$, Adv. Mater. 24, 5581 (2012). 
[69] F. Qu, C. Zhang, R.-R. Du, and L. Lu, Coexistence of Bulk and Surface Shubnikov-de Haas Oscillations in $\mathrm{Bi}_{2} \mathrm{Se}_{3}$, J. Low Temp. Phys. 170, 397 (2013).

[70] Z. Wei, Y. Rui, Z. Hai-Jun, D. Xi, and F. Zhong, FirstPrinciples Studies of the Three-Dimensional Strong Topological Insulators $\mathrm{Bi}_{2} \mathrm{Te}_{3}, \mathrm{Bi}_{2} \mathrm{Se}_{3}$ and $\mathrm{Sb}_{2} \mathrm{Te}_{3}$, New J. Phys. 12, 065013 (2010).

[71] S. V. Eremeev, M. G. Vergniory, T. V. Menshchikova, A. A. Shaposhnikov, and E. V. Chulkov, The Effect of van der
Waal's Gap Expansions on the Surface Electronic Structure of Layered Topological Insulators, New J. Phys. 14, 113030 (2012).

[72] D. Xian-Qi, Z. Bao, Z. Jian-Hua, L. Yan-Hui, T. Ya-Nan, and L. Ning, Robust Surface State of Intrinsic Topological Insulator $\mathrm{Bi}_{2} \mathrm{Te}_{2} \mathrm{Se}$ Thin Films: A FirstPrinciples Study, J. Phys. Condens. Matter 24, 035502 (2012). 